\title{
CONJUGACY CLASSES OF SUBGROUPS IN $p$-GROUPS
}

\author{
ROLF BRANDL
}

(Received 3 August 1990)

Communicated by $\mathrm{H}$. Lausch

\begin{abstract}
The set $C(G)$ of conjugacy classes of subgroups of a group $G$ has a natural partial order. We study $p$-groups $G$ for which $C(G)$ has antichains of prescribed lengths.
\end{abstract}

1991 Mathematics Subject Classification (Amer. Math. Soc.): 20 D 15, 20 D 30, 20 D 60.

\section{The results}

In recent years, there has been a considerable interest in the set $C(G)$ of all conjugacy classes [S] of subgroups $S$ of a group $G$ (see [2], [3] and the references mentioned there). This set $C(G)$ has a natural partial order defined as follows. A class $\left[S_{1}\right]$ is smaller than the class $\left[S_{2}\right]$ if and only if at least one element in $\left[S_{1}\right]$ is contained in an element of $\left[S_{2}\right]$ or, equivalently, if some conjugate of $S_{1}$ is contained in $S_{2}$. Even for relatively large groups, the poset $C(G)$ is quite small, a feature that is not shared by the lattice of all subgroups of a group $G$.

In this paper we study some order-theoretic properties of the poset $C(G)$ and investigate its influence on the group $G$. More precisely, we are interested in the Dilworth number of $C(G)$, that is, the maximum possible cardinality of an antichain in $C(G)$.

Definition. Let $G$ be a group. The Möbius-width $w_{c}(G)$ is the maximum number $t$ of subgroups $S_{1}, \ldots, S_{t}$ of $G$ with the property that no $S_{i}$ is conjugate to any subgroup of $S_{j}$ for every $j \neq i$ (if there is no such $t$,

(C)1992 Australian Mathematical Society 0263-6115/92\$A2.00+0.00 
then we set $w_{c}(G)=\infty$. Moreover, if $G$ is the trivial group, then we define $w_{c}(G)=0$ ).

An important result of Landau [5] states that for every given positive integer $n$, there exist only finitely many finite groups with precisely $n$ conjugacy classes of elements. A similar result for the width was shown in [1]: for every $n>1$ there exist only finitely many finite $p$-groups whose lattice of all subgroups has Dilworth number $n$.

For the Möbius width, an analogous result is not true in general, as for example, all dihedral groups $G$ of 2-power order satisfy $w_{c}(G)=3$. For $n>3$, however, we have the following finiteness theorem:

THEOREM A. For every $n>3$ there exist only finitely many primes $p$ and finitely many p-groups $G$ satisfying $w_{c}(G)=n$.

Clearly, every antichain of normal subgroups (with containment as the inclusion relation) forms an antichain in $C(G)$, and hence a noncyclic $p$ group $G$ must satisfy $w_{c}(G) \geq p+1$. An an illustration of Theorem A, we determine $p$-groups of small Möbius width.

Theorem B. Let $G$ be a finite p-group with $w_{c}(G)=p+1$. Then one of the following occurs:

(a) $p=2$ and $G$ is a dihedral, (generalized) quaternion or a quasidihedral group,

(b) $p>2$ and $G \cong C_{p} \times C_{p}$ or $G$ is nonabelian of order $p^{3}$ and exponent $p^{2}$.

The next possible value for the Dilworth number of the subgroup lattice of a finite $p$-group is $2 p$ (see [1]). For the Möbius width, the next following value is smaller.

THEOREM C. Let $G$ be a finite p-group of Möbius width $p+2$. Then one of the following occurs:

(a) $p=2$ and $G \cong C_{2} \times C_{4}$ or $G \cong\left\langle a, b \mid a^{8}=b^{2}=1, a^{b}=a^{5}\right\rangle$;

(b) $p \geq 3$ and $G$ is nonabelian of order $p^{3}$ and exponent $p$;

(c) $p \geq 3$ and $G \cong\langle a, b, c| a^{p}=b^{p}=c^{p^{2}}=[b, c]=1, b^{a}=b c^{s p}, c^{a}=$ $c b\rangle$ where $s=1$ or $s$ is a quadratic nonresidue $\bmod p$. If $p=3$, then we have to add the group $G \cong\langle a, b, x| a^{9}=b^{3}=[a, b]=1,[a, x]=$ $b,[b, x]=a^{3}, x^{3}=a^{3}$.

We say that a conjugacy class in $C(G)$ is of type $H$ for some group $H$, if all of its members are isomorphic to $H$. The length of a conjugacy class 
is the number of its members, the cyclic group of order $n$ will be denoted by $C_{n}$. All further unexplained notation can be found in [4]; moreover, all groups considered in this paper are finite.

\section{Finitely many $p$-groups}

This section is devoted to a proof of Theorem A. But first, we introduce some notation to facilitate the exposition.

Definition. Let $G$ be a group. A collection $S_{1}, \ldots, S_{t}$ of subgroups of $G$ is called an antichain of $G$ with respect to conjugacy (for short, a $c$-antichain) if for all indices $i, j$ with $i \neq j, S_{i}$ is not conjugate to any subgroup of $S_{j}$.

Thus, the Möbius width $w_{c}(G)$ of a group $G \neq 1$ is nothing else but the maximum taken over all cardinalities $t$ of c-antichains in $G$.

The following elementary result will be used without further mention.

LEMMA 1. Let $N$ be a normal subgroup of the group $G$. Then $w_{c}(G / N) \leq$ $w_{c}(G)$.

In the course of our investigations, we shall frequently consider c-antichains in $G$ that are contained in some normal subgroup $N$ of $G$.

Definition. Let $N$ be a normal subgroup of the group $G$. Then we define $w_{c}^{G}(N)$ to be the maximum over the lengths of all c-antichains in $G$, consisting of subgroups contained in $N$.

Clearly, we have $w_{c}^{G}(N) \leq w_{c}(G)$ and $w_{c}^{G}(G)=w_{c}(G)$.

For the proof of Theorem A, we first investigate certain abelian normal subgroups of $G$ and their connections to $w_{c}(G)$.

Lemma 2. Let $G$ be a p-group and assume that $G$ possesses a normal subgroup $N$ of exponent $p$ and order $p^{a}$, say. Then $a \leq w_{c}(G)$.

Proof. Let $1=N_{0} \leq N_{1} \leq \cdots \leq N_{a}=N$ be part of a chief series of $G$. For $1 \leq i \leq a$, choose $x_{i} \in N_{i} \backslash N_{i-1}$. Then all $G$-conjugates of $x_{i}$ belong to $N_{i} \backslash N_{i-1}$. Moreover, all $x_{i}$ are of order $p$ and hence $\left\langle x_{1}\right\rangle, \ldots,\left\langle x_{a}\right\rangle$ forms a c-antichain in $G$.

The next preparatory result provides some information on the exponent of abelian normal subgroups of $G$. 
LemMa 3. Let $N$ be an noncyclic abelian normal p-subgroup of a group G. If $\exp (N)=p^{a}$, then $a / 2 \leq w_{c}^{G}(N)$.

Proof. As $N$ is noncyclic, there exists a direct summand $A=\langle x\rangle \oplus\langle y\rangle$ of $N$ such that $o(x)=p^{a}$ and $o(y)=p^{b}$ with $1 \leq b \leq a$.

Case 1. $b \geq a / 2$. For $0 \leq i \leq a / 2$, set $S_{i}=\left\langle x^{p^{i}}, y^{p^{b-i}}\right\rangle$. Then $S_{i} \cong C_{p^{a-i}} \times C_{p^{i}}$, and hence the $S_{i}$ are pairwise nonisomorphic. As all $S_{i}$ are of the same order, they clearly form a c-antichain and the result follows here.

Case 2. $b<a / 2$. For $0 \leq i \leq a-b$, consider the cyclic subgroups $T_{i}$ of $N$, defined by $T_{i}=\left\langle x^{p^{i}} y\right\rangle$. Then $\left|T_{i}\right|=p^{a-i}$ for all $i$. If $T_{i}^{g} \leq T_{j}$ for some $g \in G$ and some $i \neq j$, then we must have $T_{i}^{g} \leq T_{j}^{p}$, because $T_{j}$ is cyclic. As $N^{p}$ is normal in $G$, this implies $T_{i} \leq\left(T_{j}^{p}\right)^{g^{-1}} \leq N^{p} \leq \Phi(N)$. But this contradicts the fact that $\langle y\rangle$ is a direct summand of $N$. Hence, $T_{0}, \ldots, T_{a-b}$ forms a c-antichain in $G$ and we have $w_{c}^{G}(N) \geq a-b>a / 2$ as claimed.

Proof of Theorem A. First, note that $n=w_{c}(G) \geq w\left(G / G^{\prime}\right) \geq p+1$, so $p \leq n-1$ and there are only finitely many primes $p$. If all abelian normal subgroups of $G$ are cyclic, then by [4, p. 304], either $G$ is a 2-group of maximal class and hence $w_{c}(G)=3<n$, or $G \cong\langle a, b| a^{2^{m}}=b^{2}=1, a^{b}=$ $\left.a^{1+2^{m-1}}\right\rangle$. But in the latter case, we have $G / G^{\prime} \cong C_{2} \times C_{2^{m-1}}$ and so [1] implies $n=w_{c}(G) \geq w\left(G / G^{\prime}\right) \geq m+1$.

Now assume that $G$ contains a noncyclic abelian normal subgroup $N$, say. We may take $N$ maximal with these properties, and so we have $N=C_{G}(N)$. First, Lemma 2, applied to $\Omega_{1}(N)$ yields that the rank $r_{p}(N)$ of $N$ satisfies $r_{p}(N) \leq w_{c}^{G}(N) \leq n$. Moreover, Lemma 3 yields that $\exp (N)$ divides $p^{2 n}$. Thus, the order of $N$ is bounded by some function of $n$. Moreover, $G / N=G / C_{G}(N)$ embeds into $\operatorname{Aut}(N)$ and hence there are only finitely many possibilities for the order of $G$. (Indeed, from [4, page 302], we can deduce an explicit upper bound for the order of $G$.) The result follows.

\section{3. $p$-groups of small order}

In this section, we determine the posets $C(G)$ and their Dilworth number for $p$-groups $G$ of order $\leq p^{4}$. For the proof of Theorems B and $\mathrm{C}$, it is sufficient to derive lower bounds for $w_{c}(G)$ in a number of cases. To 
facilitate the exposition, we do not attempt to derive the exact value here, but rather present a somewhat better bound without proof.

First, we recall some easy facts on the width of abelian groups.

LEMMA 4 ([1]). Let $p$ be a prime.

(a) We have $w_{c}\left(C_{p} \times C_{p}\right)=p+1$ and $w_{c}\left(C_{p} \times C_{p^{2}}\right)=2 p$.

(b) If $G$ is a noncyclic abelian p-group not mentioned in (a), then we have $w_{c}(G) \geq 3 p-1$.

The proof of the following simple result on groups of order $p^{3}$ illustrates the basic method that we shall use several times in this section. During the description of $C(G)$, if we state, without further comment, that a conjugacy class of subgroups contains several others, it is tacitly understood that those listed are the only ones with this property. This then determines the poset $C(G)$ and $w_{c}(G)$ can be read off. The reader is encouraged to draw his own pictures of $C(G)$ as an amusing exercise. Throughout the remainder of this section, $p$ will denote an odd prime.

LEMMA 5. Let $G$ be a nonabelian group of order $p^{3}$.

(a) If $\exp (G)=p^{2}$, then $w_{c}(G)=p+1$.

(b) If $\exp (G)=p$, then $w_{c}(G)=p+2$.

Proof. (a) Here, we start with the maximal subgroups of $G$. Indeed, $G$ contains precisely $p$ maximal subgroups of type $C_{p^{2}}$, each of which contains exactly one subgroup of order $p$, namely $G^{p}=Z(G)$ which forms a conjugacy class of length 1 . The remaining maximal subgroup $\Omega_{1}(G)$ contains $Z(G)$ and $p$ further groups of order $p$ that form a single conjugacy class. Thus, $w_{c}(G)=p+1$.

(b) Here, $G$ has $p^{2}+p+1$ subgroups of order $p$, one of them being $Z(G)$. All others are nonnormal and so they fall into $p+1$ conjugacy classes of length $p$. The centre $Z(G)$ belongs to every maximal subgroup of $G$ and each of the remaining classes of type $C_{p}$ is contained in precisely one maximal subgroup of $G$, their centraliser. Thus, $w_{c}(G)=p+2$.

Now consider groups of order $p^{4}$ where $p$ is odd. From the notation of $[4$, page $346 \mathrm{f}$.$] , we indicate the groups of order p^{4}$ by their numbers, so $G_{1}, \ldots, G_{5}$ are abelian and for obvious reasons, we do not insist on an ordering of the isomorphism types here. Moreover, $G_{6}=\langle a, b| a^{p^{3}}=$ $\left.b^{p}=1, a^{b}=a^{1+p^{2}}\right\rangle$ and $G_{9}$ corresponds to the case $s=1$ while $G_{10}$ is the group where $s$ is a nonsquare $\bmod p$. For $p=3$, there is an extra group of order $3^{4}$ (see $\left[4\right.$, p. 349]) which we will denote by $G_{\mathrm{ex}}$. Thus, $G_{\mathrm{ex}}=\left\langle a, b, x \mid a^{9}=b^{3}=[a, b]=1,[a, x]=b,[b, x]=a^{3}, x^{3}=a^{3}\right\rangle$. 
Five of the nonabelian groups are very easy to deal with.

LemMa 6. Let $p \geq 3$ and let $G$ be one of the following groups: $G_{6}, G_{7}, G_{8}, G_{11}, G_{14}$. Then $w_{c}(G) \geq 2 p$.

More explicitly, we have: $w_{c}\left(G_{6}\right)=2 p, w_{c}\left(G_{7}\right) \geq 2 p+1, w_{c}\left(G_{8}\right) \geq$ $p^{2}+p+1, w_{c}\left(G_{11}\right) \geq p^{2}+p+1$ and $w_{c}\left(G_{14}\right) \geq p^{2}+p+1$.

Proof. The groups $G_{6}$ and $G_{7}$ map onto $C_{p} \times C_{p^{2}}$ and the remaining ones map onto $C_{p} \times C_{p} \times C_{p}$. The result follows from Lemma 4 . The proof of the remaining statements is omitted.

Now we consider the groups of smallest Möbius width.

LeMMA 7. Let $p \geq 3$. Then $w_{c}\left(G_{9}\right)=w_{c}\left(G_{10}\right)=p+2$.

Proof. First, note that $G$ is a split extension of the normal subgroup $N=\langle y, z\rangle \cong C_{p} \times C_{p^{2}}$ by the cyclic group $\langle x\rangle$ of order $p$. Moreover, $G$ is of maximal class and hence $\Omega_{1}(N)=\left\langle y, z^{p}\right\rangle$ is the unique normal subgroup of order $p^{2}$ in $G$.

We first deal with the case $p \geq 5$. Then $G$ is regular. As $\exp (G)=p^{2}$, we see that $\Omega_{1}(G)=\left\langle x, y, z^{p}\right\rangle$ is nonabelian of order $p^{3}$ and exponent $p$. Now there are precisely $p^{2}$ cyclic subgroups of order $p^{2}$ in $G$. By the above, none of them is normal in $G$, and hence each has precisely $p$ conjugates. Thus, there are $p$ classes of type $C_{p^{2}}$. Also, the normaliser of a cyclic subgroup of order $p^{2}$ in $G$ is a group of order $p^{3}$ and exponent $p^{2}$ (indeed, one of them is abelian, namely $N$, and all others are nonabelian).

Now there are three "types" of elements of order $p$, namely the central ones $z^{p}$, the abelian ones contained in $\Omega_{1} \backslash\left\langle z^{p}\right\rangle$ and the ones "outside". An inspection of these shows that there are three classes of type $C_{p}$ with representatives $\left\langle z^{p}\right\rangle,\langle y\rangle$ and $\langle x\rangle$. In fact, among the $p$ maximal subgroups of exponent $p^{2}$, the $p-1$ nonabelian ones contain the class with representative $\langle y\rangle$ and $N$ contains $\left\langle z^{p}\right\rangle$.

Next, consider subgroups of type $C_{p} \times C_{p}$. Each of the above normal subgroups of exponent $p^{2}$ contains precisely one characteristic subgroup of order $p^{2}$, their $\Omega_{1}$. As this is normal in $G$, it must coincide with $\Omega_{1}(N)$.

Now the remaining maximal subgroup is $\Omega_{1}(G)$, which is of exponent $p$. This clearly contains $\Omega_{1}(N)$ which is the only one which is normal in $G$. As it contains precisely $p$ further such subgroups, they are all conjugate in $G$. Thus, we have determined $C(G)$ and finally, we see that $w_{c}(G)=p+2$.

The case $p=3$ is similar.

The extra group $G_{\text {ex }}$ has a "similar" Moebius-poset and we omit the proof of the following result. 
Lemma 8. Let $p=3$. Then $w_{c}\left(G_{\mathrm{ex}}\right)=5=p+2$.

The remaining groups can be dealt with by using similar methods and we only present the result.

LEMMA 9. (a) We have $w_{c}\left(G_{12}\right) \geq 2 p+1$ if $p=3$, and $w_{c}\left(G_{12}\right) \geq 2 p+2$ if $p \geq 5$.

(b) We have $w_{c}\left(G_{13}\right) \geq 2 p+2$ if $p=3$, and $w_{c}\left(G_{13}\right) \geq 2 p+3$ if $p \geq 5$.

(c) We have $w_{c}\left(G_{15}\right) \geq 2 p+2$ if $p=3$, and $w_{c}\left(G_{15}\right) \geq 2 p+3$ if $p \geq 5$.

Part b) of Theorem B follows from the results of this section, because all noncyclic groups of order at least $p^{4}$ have Möbius width $\geq p+2$.

\section{Larger groups ?}

The proof of Theorem $\mathrm{C}$ is by showing that all noncyclic $p$-groups of Möbius width $\leq p+2$ are of order $\leq p^{4}$. Now in Section 3, we have determined all such groups of this order, and so, by way of contradiction, we may assume that there exists a noncyclic $p$-group $H$ of order $\geq p^{5}$ satisfying $w_{c}(H) \leq p+2$. Clearly then, there exists a noncyclic factor group $\widetilde{G}$ of $H$ with the following properties: $|\widetilde{G}|=p^{5}$ and $w_{c}(\widetilde{G}) \leq p+2$. Obviously, $\widetilde{G}$ is nonabelian, so let $M$ be a minimal normal subgroup of $\tilde{G}$ contained in $\widetilde{G}^{\prime}$. Thus, $M \leq \widetilde{G}^{\prime} \cap Z(\widetilde{G})$, and hence $G=\widetilde{G} / M$ has a nontrivial Schur multiplicator. By Section 3, we have $G \cong G_{9}, G_{10}$ or $G_{\text {ex }}$. We shall keep this notation for the rest of this section.

LEMMA 10. Let $G=G_{9}$ or $G_{10}$ if $p \geq 5$, or $G=G_{9}$ if $p=3$ or $G=G_{\mathrm{ex}}$. Then $w_{c}(\tilde{G}) \geq 2 p$.

Proof. We first show that $Z(\widetilde{G})$ is elementary abelian of rank two. For this, we use some information on the quotient $G$ of $\widetilde{G}$ that we know about. In all cases, $G=\widetilde{G} / M$ contains precisely $p-1$ maximal subgroups $E_{1} / M, \ldots, E_{p-1} / M$ which are nonabelian of exponent $p^{2}$ and of order $p^{3}$. For $1 \leq i \leq p-1$, we have $M \leq Z\left(E_{i}\right)$. As $E_{i} / M$ has trivial Schur multiplicator, we must have $M \cap E_{i}^{\prime}=1$. As $E_{i} / M$ is of class two, this implies $\left[E_{i}, E_{i}^{\prime}\right] \leq M \cap E_{i}^{\prime}=1$, and hence $E_{i}$ is of class two. As $M \leq Z\left(E_{i}\right)$, this shows that $M \times E_{i}^{\prime} \leq Z\left(E_{i}\right)$. Let $Z / M=Z(\widetilde{G} / M)$, so $Z / M$ is of order p. Then $M E_{i}^{\prime} / M \leq Z(\widetilde{G} / M)=Z / M$, and hence we have $M \times E_{i}^{\prime}=Z$. As $M \times E_{i}^{\prime} \leq Z\left(E_{i}\right)$ for $i=1,2$, and $E_{1} E_{2}=\widetilde{G}$, we get $Z \leq Z(\widetilde{G})$ and our claim is proved. 
We now show that $w_{c}(\widetilde{G}) \geq 2 p$. Indeed, $Z$ contains $p$ normal subgroups $C_{1}, \ldots, C_{p}$ of order $p$, distinct from $M$. Thus, we have $C_{j} M / M=$ $Z(\widetilde{G} / M)$ for all $j$. Now let $D_{1} / M, \ldots D_{p} / M$ be representatives of the $p$ classes of cyclic subgroups of order $p^{2}$ in $\tilde{G} / M$. As these are pairwise incomparable with $Z(\widetilde{G} / M)$, the subgroups $C_{1}, \ldots, C_{p}, D_{1}, \ldots D_{p}$ of $\widetilde{G}$ form a c-antichain in $\widetilde{G}$, and we therefore have $w_{c}(\widetilde{G}) \geq 2 p$.

We now deal with the exceptional case when $p=3$.

Lemma 11. Let $G=G_{10}$ and assume that $p=3$ and $s=-1$. Then $w_{c}(\tilde{G}) \geq 6$.

Proof. First, $\widetilde{G} / M$ is of maximal class, and hence it has precisely one normal subgroup of order 3. Moreover, by Lemma $7, G=\widetilde{G} / M$ has a cantichain $S_{1} / M, \ldots, S_{5} / M$ of cyclic subgroups of order 3, precisely one of them is normal in $G$, say $S_{1} / M$. Let $W / M$ be the maximal subgroup of $G$ isomorphic to $C_{3} \times C_{9}$. We show that one of the following conditions hold:

(a) $W$ contains two distinct characteristic subgroups or order 9; or

( $\beta) W$ contains a subgroup $U$ of order 9 with $M \cap U=1$.

In both cases, it follows that $w_{c}(\widetilde{G}) \geq 6$. Indeed, if $(\alpha)$ holds, then we can choose a characteristic subgroup $C$ of order 9 of $W$ with $C \neq S_{1}$. Then $C$ is normal in $\widetilde{G}$ and $S_{1}, \ldots, S_{5}, C$ is a c-antichain in $\widetilde{G}$. In the situation ( $\beta$ ), no conjugate of $U$ contains $M$. As all conjugates of $S_{1}, \ldots, S_{5}$ contain $M$, we have that $S_{1}, \ldots, S_{5}, U$ is a c-antichain and in both cases, it follows that $w_{c}(\widetilde{G}) \geq 6$.

We now prove the above claim. Indeed, if $W$ contains an abelian subgroup $A$ of rank $\geq 3$, then we clearly can choose $U$ as a suitable subgroup of $A$ and we have $(\beta)$. If $W$ is abelian, there are two more cases: if $W \cong$ $C_{3} \times C_{27}$, we have $(\alpha)$ and if $W \cong C_{9} \times C_{9}$, we have $(\beta)$. So let $W$ be nonabelian. As $W / M \cong C_{3} \times C_{9}$, the list of all groups of order $3^{4}$ gives two more possibilities for $W$. If $W \cong\left\langle a, b \mid a^{27}=b^{3}=1, a^{b}=a^{10}\right\rangle$, we have $\Omega_{1}(W) \neq W^{3}$ and $(\alpha)$ holds. Finally, in $W \cong\langle a, b| a^{9}=b^{9}=$ 1, $\left.a^{b}=a^{4}\right\rangle$, we must have $M=W^{\prime}=\left\langle a^{3}\right\rangle$ and $U:=\langle b\rangle$ has trivial intersection with $M$. The result follows.

By the remarks on the beginning of this section, there are no noncyclic $p$-groups of order $\geq p^{5}$ and Möbius width $\leq p+2$, and so parts (b) and (c) of Theorem $\mathrm{C}$ are proved. 


\section{The case $p=2$}

In this final section, we consider groups of even order. Here, the situation is quite different as the 2-groups $G$ of maximal class satisfy $w_{c}(G)=3$. However, it turns out that there are only finitely many additional examples.

Proposition 12. Let $G$ be a 2-group satisfying $w_{c}(G) \leq 4$. Then one of the following holds:

(i) $G$ is cyclic;

(ii) $G \cong C_{2} \times C_{4}$;

(iii) $G / G^{\prime} \cong C_{2} \times C_{2}$ and $G$ is of maximal class;

(iv) $G \cong\left\langle a, b \mid a^{8}=b^{2}=1, a^{b}=a^{5}\right\rangle$.

Proof. Let $G$ be a counterexample of least possible order. Lemma 4 implies that $G$ is nonabelian. If $G / G^{\prime} \cong C_{2} \times C_{2}$, then [4, p. $339 \mathrm{f}$.] shows that we have (iii). By Lemma 4 again, we have $G / G^{\prime} \cong C_{2} \times C_{4}$. Let $M$ be a minimal normal subgroup of $G$ contained in $G^{\prime}$. Then $M \leq G^{\prime} \cap Z(G)$ and hence $G$ is a covering group of $Q:=G / M$. As $w_{c}(Q) \leq w_{c}(G)$, induction applies. Clearly, $Q$ cannot be of type (i). If $Q$ is of type (iii), then $G$ is of type (iii). As the group mentioned in (iv) has a trivial Schur multiplicator, this case cannot occur here, so let $Q$ be of type (ii). An inspection of all groups of order 16 shows that we are left with three possibilities. First, $G$ may well be of type (iv). The next possibility would be

$$
G \cong\left\langle a_{1}, a_{2}, x \mid a_{1}^{4}=a_{2}^{2}=x^{2}=\left[a_{1}, a_{2}\right]=\left[a_{2}, x\right]=1, a_{1}^{x}=a_{1} a_{2}\right\rangle .
$$

Here, $\Omega_{1}(G) \cong C_{2} \times C_{2} \times C_{2}$ contains seven Klein groups each of which has at most two conjugates. As $G$ contains cyclic subgroups of order 4 , we have $w_{c}(G) \geq 5$ here. The last possibility is $G \cong\left\langle a, b \mid a^{4}=b^{4}=1, a^{b}=a^{3}\right\rangle$. Here, $\Omega_{1}(G)=\left\langle a^{2}, b^{2}\right\rangle$ is of order 4 , and hence $G$ contains six cyclic subgroups of order 4 . At least one of them is normal and the remaining ones have at most two conjugates. These classes, together with $\Omega_{1}(G)$, form a c-antichain of length 5 and our result follows.

\section{References}

[1] R. Brandl, 'The Dilworth number of subgroup lattices', Arch. Math. 50 (1988), 502-510.

[2] T. Hawkes and I.M. Isaacs, 'On the poset of $p$-subgroups of a p-solvable group', J. London Math. Soc. (2) 38 (1988), 77-86. 
[3] T. Hawkes, I.M. Isaacs and M. Özaydin, 'On the Möbius function of a finite group', Rocky Mountain J. Math. 19 (1989), 1003-1034.

[4] B. Huppert, Endliche Gruppen I (Springer-Verlag, Berlin, 1967).

[5] E. Landau, 'Über die Klassenzahl der binären quadratischen Formen von negativer Discriminante', Math. Ann. 56 (1903), 671-676.

Mathematisches Institut

Am Hubland 12

D-8700 Würzburg

West Germany 